\title{
Three-body interaction effects on the ground state of one-dimensional anyons
}

\author{
J. Arcila-Forero, R. Franco, and J. Silva-Valencid* \\ Departamento de Física, Universidad Nacional de Colombia, A. A. 5997 Bogotá, Colombia.
}

(Dated: March 19, 2019)

\begin{abstract}
We investigated a one-dimensional system of anyons that interact with each other under a local three-body term. Using a fractional Jordan-Wigner transformation, we arrived at a modified BoseHubbard model, which exhibits gapped and gapless phases. We built the phase diagram of the system fixing the hopping parameter or the statistics, showing the evolution of the critical points, which were estimated with von Neumann block entropy. A superfluid to Mott insulator quantum phase transition with one particle per site can be driven by the statistics or the interaction. Specifically, we show that for larger angles there is a finite critical value of the interaction at which the Mott phase appears. Also, we found that the critical angles increase with the hopping. Diverse gapless phases were observed away from the pseudo-fermion limit.
\end{abstract}

\section{INTRODUCTION}

Commonly, particles in quantum theory are classified into two types, bosons and fermions, depending on their particle exchange characteristics. For two bosons, the wavefunction remains invariant under particle exchange, whereas the exchange of two fermions leads to a multiplicative factor -1 in the wavefunction. Physicists have proposed a third class of particles with nontrivial exchange statistics, anyons, particles carrying fractional statistics that interpolate between bosons and fermions [1 3]. For two anyons under particle exchange, the wave function acquires a fractional phase $e^{i \theta}$, giving rise to fractional statistics with $0<\theta<\pi$. It was quickly found that relevant quantum phenomena can be explained in terms of these new particles, the fractional quantum Hall effect [4 7] being the best known, and nowadays it is believed that anyons will be important in condensed matter physics and topological quantum computation 8,10$]$.

The study of anyons was restricted for many years to two-dimensional systems. However, with Haldane's definition of fractional statistics, it was generalized to arbitrary dimensions [3]. Anyons in one dimension (1D) have been widely studied, their commutation relations [11, dispersion relations and the dependence between generalized exclusion statistics and the anyonic angle [12, 13], correlation functions in the low-momentum regime 14, the dynamical properties in a weak harmonic trap [15], the ground state properties of hard-core anyons [16], and mixtures of anyons under an external trap [17] having been found. Also, it has been observed that the excitations of some one-dimensional models can be explained in terms of anyons [18, 19].

The creation and manipulation of physical setups that display fractional statistics is a huge challenge for experimental physicists, although several proposals have been made [20 25]. One of the most popular approaches for observing fractional physics is to confine

\footnotetext{
* jsilvav@unal.edu.co
}

ultracold bosonic atoms in an optical lattice, whose requirements have been considerably changed and simplified recently [26 29]. The anyon-Hubbard model, which takes into account the kinetic energy of the anyons and the local two-body repulsion between them, arises from the above proposals. The ground state and the phase diagrams as a function of the tunneling, the local interaction, and the statistics have been studied using mean field theory and the density matrix renormalization group method 26, 27, 30, 31]. In others studies, the quasimomentum distribution [32], the one-body reduced density matrix in a harmonic potential [33], and a nontrivial topological Haldane phase were explored [34].

In the experimental setups of cold atoms using optical lattices, it is possible to tune the tunneling parameter of particles in a lattice, the interaction strength through Feshbach resonance, the density of the particles, and the dimensionality, and for this reason they have emerged as unusual laboratories for the realization of boson- and Fermi-Hubbard models, as well as for observing phase transitions without the intrinsic uncertainty posed by materials [35, 36]. Also, these setups have allowed the observation of many-body interaction effects [37, 38], stimulating new experimental proposals and theoretical calculations about the physical properties of bosonic systems with relevant three-body interactions between particles 39 42].

An unexplored problem consists of considering delocalized anyons in a one-dimensional optical lattice under local three-body interactions. This problem is interesting because the three-body interactions between spinless or spinor bosons cannot generate a Mott insulator state with one particle per site and also change the phase diagram. On the other hand, it has been shown that the anyonic statistics localize the particles, and this can induce a quantum phase transition in systems with two-body interactions. In the present paper, we study the interplay between the above phenomena and write a Hamiltonian with two terms: the local three-body interaction and the kinetic energy. Using a fractional Jordan-Wigner transformation, this Hamiltonian was mapped to a modified Bose-Hubbard Hamiltonian with the tunneling depending on the local density and local three-body interactions, 
which will be studied numerically. The ground state can be gapped or gapless, in accordance with our density matrix renormalization group results. For a global density of one particle per site, we show that the interplay of statistics and the three body interactions leads to a Mott insulator state away from the pseudo-fermion limit. Note that at the above limit a Mott insulator state was found in the absence of interactions 27]. We build two phase diagrams, the chemical potential as a function of the statistical angle for a fixed hopping parameter and the chemical potential versus the hopping for a fixed anyonic angle. Although a complete study of the superfluid regions is beyond the scope of this paper, our results indicate that for non-integer densities smaller than one, the ground state is a superfluid that does not depend on statistics. After that, a paired phase appears even at the pseudo-fermion limit, this being an important difference between two- and three-body results. Also, we obtained a superfluid phase for densities larger than one at the pseudo-fermion limit. We expect that these facts will be observed in the near future when recent proposals for creating an anyon system and obtaining a regime where the three-body interaction domain is realized. We would like to point out that a prior report on the interplay between many-body effects and anyonic statistics has been made [43].

Our study is organized in the following way: The anyon-Hubbard model with local three-body interactions is explained in Sec. IA. Using the von Neumann block entropy and the excitation gap in sec. [B] we distinguish the quantum phases of the model and draw the phase diagrams of the model. Finally, our main findings and final comments appear in Sec. [IC]

\section{A. Model}

In one dimension, the operators of creation $\left(a_{j}^{\dagger}\right)$ and annihilation $\left(a_{j}\right)$ of an anyon at site $j$ satisfy the following commutation relations:

$$
\begin{gathered}
a_{j} a_{k}^{\dagger}-e^{-i \theta \operatorname{sgn}(j-k)} a_{k}^{\dagger} a_{j}=\delta_{j k}, \\
a_{j} a_{k}-e^{i \theta \operatorname{sgn}(j-k)} a_{k} a_{j}=0,
\end{gathered}
$$

where $\theta$ denotes the statistical phase, and the sign function (multistep function) is $\operatorname{sgn}(j-k)= \pm 1$ for $j>k$ and $j<k$, and $\operatorname{sgn}(j-k)=0$ for $j=k$. Note that, two particles on the same site reproduce the ordinary bosonic commutations relations.

The quantum phases of a set of soft-anyons is a subject of great interest at this moment, and they come from the interplay between the hopping of the carries throughout the lattice and the interactions. In the present paper, we consider repulsive local three-body interactions, and the
Hamiltonian is given by

$$
\begin{aligned}
H= & -t \sum_{j}^{L-1}\left(a_{j}^{\dagger} a_{j+1}+\text { h.c. }\right) \\
& +\frac{W}{6} \sum_{j}^{L} n_{j}\left(n_{j}-1\right)\left(n_{j}-2\right),
\end{aligned}
$$

$t>0$ being the tunneling amplitude connecting two neighboring sites, $W$ the on-site interaction, $L$ the length of the lattice, and $n_{j}$ the number operator. The first term in the Hamiltonian (2) is the kinetic energy with strength $t$, and the second term stems from the short-range interaction between three particles. Our unit of energy will be $W=1$ unless otherwise specified.

The fractional version of the Jordan-Wigner transformation

$$
a_{j}=b_{j} \exp \left(i \theta \sum_{i=1}^{j-1} n_{i}\right),
$$

defined by Keilmann et al. establishes an exact mapping between anyons and bosons in one-dimension [26]. Here, the operator $b_{j}$ describes spinless bosons, which satisfy $\left[b_{j}, b_{i}^{\dagger}\right]=\delta_{j i}$ and $\left[b_{j}, b_{i}\right]=0$. Note that the representation of the number operator in terms of anyon or boson operators is the same $\left(n_{i}=a_{i}^{\dagger} a_{i}=b_{i}^{\dagger} b_{i}\right)$.

Applying the anyon-boson mapping (3) to the anyonHubbard Hamiltonian with three-body interaction (2), we obtain a Hamiltonian in terms of boson operators, which allows us to explore the ground state but also establishes a link between the anyon-Hubbard Hamiltonian and its possible experimental realization in cold atom setups. Therefore, the bosonic version of the anyon-Hubbard Hamiltonian with three-body interaction is given by:

$$
\begin{aligned}
H= & -t \sum_{j}^{L-1}\left(b_{j}^{\dagger} b_{j+1} e^{i \theta n_{j}}+\text { h.c. }\right) \\
& +\frac{W}{6} \sum_{j}^{L} n_{j}\left(n_{j}-1\right)\left(n_{j}-2\right) .
\end{aligned}
$$

In this way, the above Hamiltonian describes bosons with the tunneling depending on the local density $t e^{i \theta n_{j}}$ for hopping processes from right to left $(j+1 \rightarrow j)$. All of the influence of the fractional statistics is concentrated in this term. If the target site $j$ is unoccupied, the hopping amplitude is simply $t$. If it is occupied by one boson, the amplitude reads $t e^{i \theta}$, for two bosons $t e^{i 2 \theta}$, and so on.

\section{B. Results}

Nowadays it is well known that quantum information tools are useful for distinguishing different quantum 


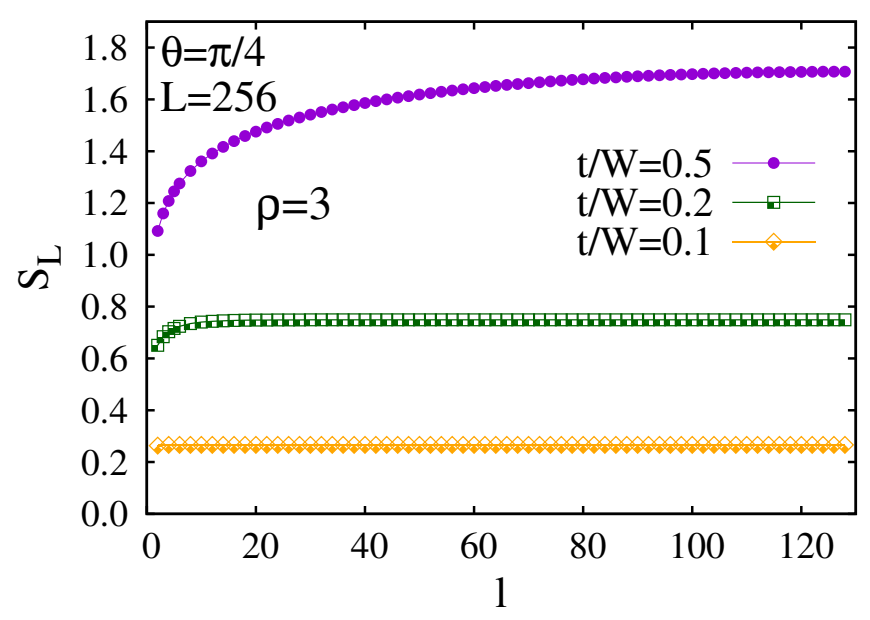

FIG. 1. The von Neumann block entropy $S_{L}(l)$ as a function of $l$ for an anyon chain with $\theta=\pi / 4, \rho=3$ and size $L=$ 256. Here we consider three different values of the hopping parameter: $t / W=0.1,0.2$, and 0.5 . Clearly, we can see that a change of state happens as the hopping grows.

phases, and also we can estimate the border or critical point that separates one region from another, although there is no "universal" measure of entanglement that works for any quantum problem [44]. Despite the relevance emphasized by many people and the possible applications of entanglement in diverse areas of physics, experiments for directly detecting and measuring the degree of entanglement of a system are challenging for science. Recently, in an experiment with atoms confined in optical lattices, it was possible to measure the quantum purity, Rényi entanglement entropy, and mutual information of a Bose-Hubbard system [45]. These results reinforced the connection between quantum information tools and the description of degenerate gases realized in cold atom setups.

Bipartite measures of entanglement are the most used. Therefore, we consider a system with $L$ sites divided into two parts. Part $A$ has $l$ sites $(l=1, \ldots, L)$, and the rest form part $B$, with $L-l$ sites. The von Neumann block entropy of block $A$ is defined by $S_{A}=-\operatorname{Tr} \varrho_{A} \ln \varrho_{A}$, where $\varrho_{A}=\operatorname{Tr}_{B} \tilde{\varrho}$ is the reduced density matrix of block $A$ and $\tilde{\varrho}=|\Psi\rangle\langle\Psi|$ the pure-state density matrix of the whole system. For a system with open boundary conditions, the behavior of the von Neumann block entropy as a function of $l$ depends on the nature of the ground state and provides information about the type of phase, because it saturates (diverges) if the system is gapped (gapless) [46]. Thus:

$$
S_{L}(l)=\left\{\begin{array}{lc}
\frac{c}{6} \ln \left[\frac{2 L}{\pi} \sin \left(\frac{\pi l}{L}\right)\right]+\Theta, & \text { critical } \\
\frac{c}{6} \ln \left[\xi_{L}\right]+\Theta^{\prime}, & \text { non critical }
\end{array}\right.
$$

$c$ being the central charge and $\xi_{L}$ the correlation length. The constants $\Theta$ and $\Theta^{\prime}$ are nonuniversal and model dependent.

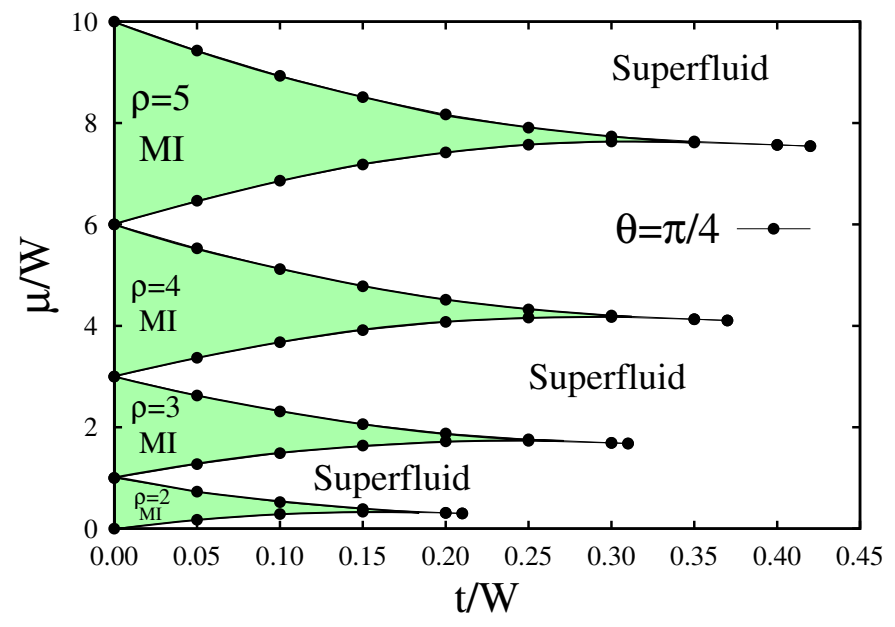

FIG. 2. Phase diagram of the anyon-Hubbard model with local three-body interactions for $\theta=\pi / 4$ and for the densities $\rho=2,3,4$ and 5 . The points correspond to extrapolations to the thermodynamic limit from DMRG data, and the lines are visual guides. MI means Mott insulator regions. The elongated form of the Mott insulator lobes suggests that the transitions are of the Kosterlitz-Thouless type, and the critical points can be calculated by means of gap-scaling analyses [47, 48] or a scaling of the Luttinger-liquid parameter [49] or the Läuchli and Kollath estimator [50].

To determine the ground-state wave function $(|\Psi\rangle)$ of a lattice with $L$ sites and $N$ particles, we used the finite-size density matrix renormalization group algorithm (DMRG) with open boundary conditions, and in order to improve the calculations, we implemented the dynamical block state selection (DBSS) protocol based on a fixed truncation error of the subsystems reduced density matrix instead of using a fixed number of preserved states in the DMRG sweeps [51]. Also, we truncated the local Hilbert space by considering only $\rho+5$ states when the density of the particles is $\rho=N / L$ [47]. With the above considerations, we obtained a discarded weight of around $10^{-10}$ or less, and the maximum number of states maintained was $m=1200$.

The evolution of the von Neumann block entropy $S_{L}(l)$ as the size of the block increases is shown in Fig. 1 for a lattice with global density $\rho=3$, statistical angle $\theta=\pi / 4$, and three different values of the hopping: $t / W=0.1, t / W=0.2$, and $t / W=0.5$. For small values of the hopping parameter, we expected that the particles would tend to localize and the entanglement would be small. This happened for $t / W=0.1$, and we observed that the von Neumann block entropy saturates very quickly and has a small value. The above behavior continues for larger values of the hopping parameter $(t / W=0.2)$, but the maximum numerical value is larger, indicating that the entanglement has increased. These results indicate that for a wide range of values of the hopping the system remains in a phase characterized by a finite correlation length, in accordance with the expression Eq. (5). However, things change for larger values. 


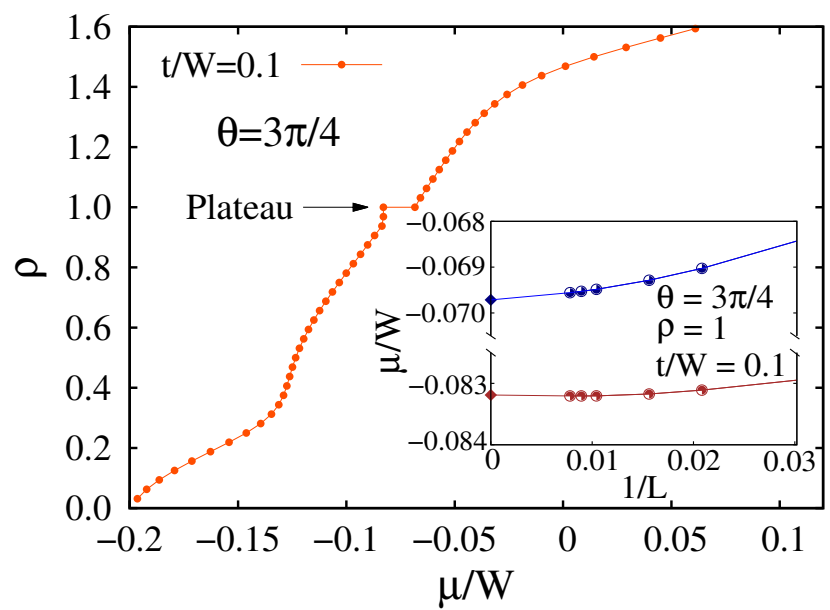

FIG. 3. Global density $\rho$ versus the chemical potential $\mu / W$ for $t / W=0.1$ and a statistical angle $\theta=3 \pi / 4$. The system exhibits a Mott plateau at integer density $\rho=1$. Inset: System size dependence on the chemical potential of anyons with three-body interactions for statistical angle $\theta=3 \pi / 4$ and $\rho=1$. The upper set data correspond to the particle excitation energy and the lower to the hole excitation energy (the lines are visual guides). The values for $1 / L=0$ (diamonds) correspond to an extrapolation to the thermodynamic limit.

For $t / W=0.5$, the von Neumann block entropy increases smoothly and tends to diverge with the block size, which characterizes a critical state. In this way, the calculation of the von Neumann block entropy allows us to distinguish between two quantum phases in the system (one critical and the other non-critical) and thus to discern the appearance of a phase transition as we increase the tunneling of the particles. This can be deduced from the fact that the entropy changes its behavior (saturate-diverge) when we increase the value of $t / W$. In this case, considering that the system density is an integer and that we are facing a non-critical phase, the results suggest that the system could be found to be in a Mott-insulator phase characterized by having a finite-correlation length. This would explain the fact that the block entropy increases and saturates rapidly at a constant value. On the contrary, in the critical region, the system could exhibit a superfluid state, characterized by a divergent behavior of the von Neumann block entropy and the delocalization of the particles along the lattice. In conclusion, for a fixed global density $\rho$, the anyon-Hubbard model with local three-body interactions exhibits a Mott insulator to superfluid transition for a finite value of $t / W$.

The phase diagram of anyons in one dimension under two-body interactions shows Mott lobes surrounded by superfluid regions, with the Mott areas decreasing as the global density grows. In particular, it was found that the critical point for a statistical angle $\theta=\pi / 4$ and a fixed global density $\rho=3$ is located at $t / U=0.172$, $U=1$ being the strength of the two-body interactions and the energy scale for this case [26, 30]. In Fig. 1] we observe that the Mott lobe for $\rho=3$ survives for values

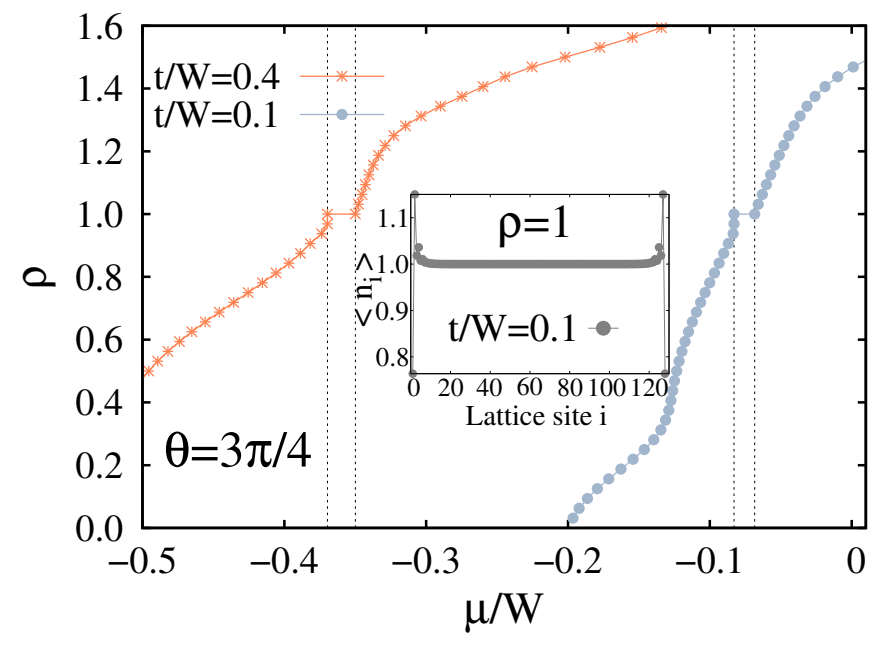

FIG. 4. Global density $\rho$ as a function of the chemical potential $\mu / W$ at the thermodynamic limit. Here, we fix the statistical angle to $\theta=3 \pi / 4$ and consider $t / W=0.1$ and $t / W=0.4$. The vertical dashed lines delimit the insulator phases. In the inset, we show the density profile along the lattice for $\theta=3 \pi / 4, t / W=0.1$ and $L=120$.

greater than $t / W=0.2$, which indicates that the Mott lobes with three-body interactions will be larger than the lobes with two-body interactions, indicating that threebody interactions generate a larger localization in the system, in accordance with previous results for bosons with three-body interactions.

We found that the Hamiltonian problem (2) exhibits Mott and superfluid states, which are characterized by the presence or the lack of an excitation gap at the thermodynamic limit. For a lattice with finite size, the excitation gap is $\Delta \mu(L)=\mu^{p}(L)-\mu^{h}(L)=E_{0}(L, N+$ $1)+E_{0}(L, N-1)-2 E_{0}(L, N)$, where $E_{0}(L, N)$ denotes the ground-state energy for $L$ sites and $N$ particles. In particular, a Mott-insulator phase is characterized by the presence of a positive gap at the thermodynamic limit, since the global density is an integer, while the superfluid phase is characterized by the fact that no gap exists at the thermodynamic limit. In Fig. 2, we show the thermodynamic limit values of the chemical potential of adding $\left(\mu^{p}\right)$ and removing $\left(\mu^{h}\right)$ a particle as a function of the hopping for a statistical angle $\theta=\pi / 4$ and different global densities $\rho$. As is well known, the gapped regions are surrounded by superfluid (gapless) ones, and we obtained that upon increasing the global density, the insulator lobes increase and the position of the critical points moves to greater values of $t / W$ in a manner similar to the boson problem $(\theta=0) 52$, but for anyons the critical points are greater than for bosons. These results indicate that the three-body interaction and the statistics favor the localization of particles and that greater kinetic energy is required to delocalize the particles and generate superfluid states. Another characteristic of Fig. 2 is the absence of the insulator region for $\rho=1$, which is due to the fact that the quantum fluctuations, hav- 


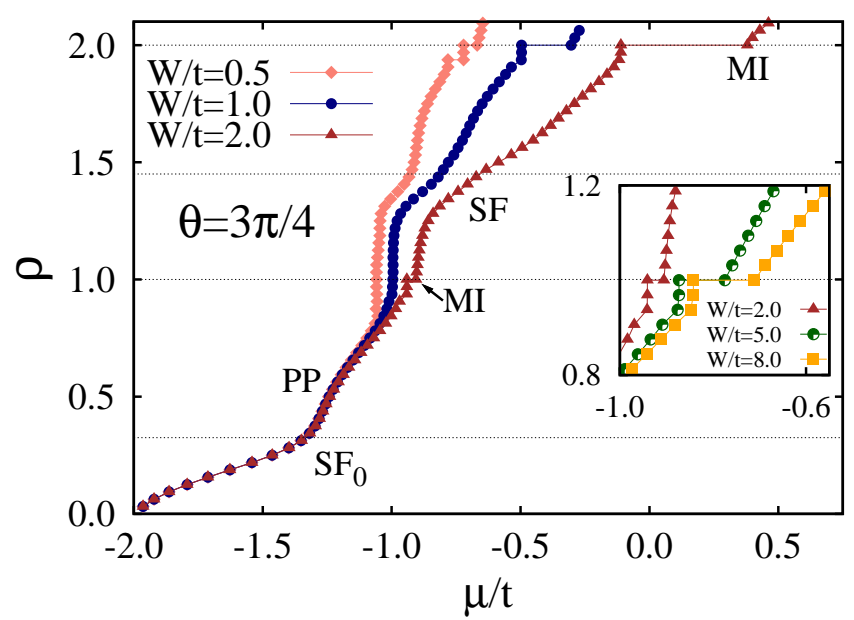

FIG. 5. Global density $\rho$ versus the chemical potential $\mu / t$ at the thermodynamic limit with a statistical angle $\theta=3 \pi / 4$ for different values $W=0.5,1.0$, and 2.0. Here the unit of energy is the hopping parameter $t=1$. A zoom of the main figure around the first plateau appears in the inset, where the three-body local interaction is $W=2.0,5.0$, and 8.0. The horizontal lines are visual guides.

ing on average only one particle per site $(\rho=1)$ and $\theta=\pi / 4$, are insufficient for generating particle localization. The elongated shape of the Mott-insulator lobes indicates that the gap closes slowly and possibly follows a Kosterlitz-Thouless formula [30]. The length of the Mott regions on the vertical axis at $t / W=0$ varies with the density of the system. It is notable that for the Boseand anyon-Hubbard models with two-body interaction, this length does not change with the density. Rather, it is constant and equal to one in the scale of $\mu / U$. When we consider three-body interactions, the length of the lobe with global density $\rho$ at $t / W=0$ is $\rho-1$ for both bosons and anyons with $\theta=\pi / 4$.

One of the main findings in the anyon-Hubbard model is that the statistics favor the localization of the particles, which is reflected in the increase in the area of the Mott lobes as the statistical angle $\theta$ tends toward $\pi$ [26]. The absence of the $\rho=1$ Mott insulator lobe in spinless or spinor bosons chains under local three-body interactions is a very well-known result, but in the present paper, our subject of study consists of anyons, and we expected that the statistical angle would generate new findings. Hence we wanted to explore if for $\theta>\pi / 4$ new insulator phases can appear. Taking into account the above and the fact that a Mott insulator state appears in the absence of interactions at the pseudo-fermion limit $(\theta=\pi)$ [27], we decided to distance ourselves from the above limit. Therefore, we fixed the statistical angle to $\theta=3 \pi / 4$ and studied the evolution of the chemical potential as the number of anyons increases. This leads us to Fig. 3, where we show the global density as a function of the chemical potential at the thermodynamic limit. Note that as we increase the overall density of the system,
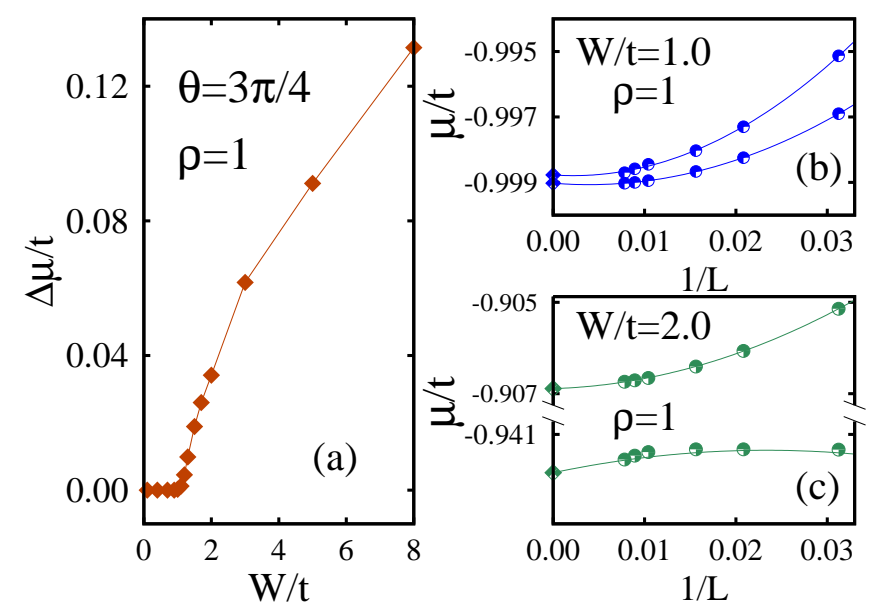

FIG. 6. Left panel: The excitation gap at the thermodynamic limit as a function of the three-body interaction for a system of anyons with $\theta=3 \pi / 4$ and global density $\rho=1$. The critical point $W_{c} / t=1.1$ separates a gapless region and a Mott insulator one. Right panel: System size dependence on the chemical potential of a system with one particle per site, $\theta=3 \pi / 4$, and local interaction $W / t=1.0$ and $W / t=2.0$ for Figures (b) and (c), respectively. In both figures, the upper set of data corresponds to the particle excitation energy and the lower one to the hole excitation energy. The points are the DMRG results, and the lines are visual guides. The values for $1 / L=0$ correspond to an extrapolation to the thermodynamic limit, using a second-order polynomial in $1 / L$.

the chemical potential increases for all the non-integer densities, but for a global density of $\rho=1$, a plateau appears, which indicates the existence of a finite gap at the thermodynamic limit for this density, given by the width of the plateau. In the inset of Fig. 3, we show the evolution of the energies for adding $\left(\mu^{p}\right)$ and removing $\left(\mu^{h}\right)$ particles versus the inverse of the lattice size for anyons with $\theta=3 \pi / 4$ and density $\rho=1$. This evolution is quadratic for $t / W=0.1$, and at the thermodynamic limit we obtain $\Delta \mu / W=\lim _{L, N \rightarrow \infty}\left[\mu^{p}(L)-\mu^{h}(L)\right]=$ 0.0135 , which corresponds to the width of the plateau and allows us to conclude that the ground state for $\theta=3 \pi / 4$ and $\rho=1$ is an insulator.

Fixing the hopping parameter to $t / W=0.1$, we can compare Figs. 2 and 3 and observe that the quantum fluctuations are very small for $\theta=\pi / 4$. Therefore, the interaction term is unimportant and the ground state is a superfluid (gapless), and the first insulator state is obtained for a global density of $\rho=2$. However, as the statistical angle increases, the particles are more localized, the effect of the effective interaction term grows, and an insulator state is obtained for $\rho=1$. The above insulator state is surrounded by gapless regions (see Fig. 3), but we note that the evolution is different for values greater than or less than $\rho=1$, and also for $\rho<1$ two diverse regions can be identified, which indicates that the increase of the statistical angle enriches the superfluid regions. 


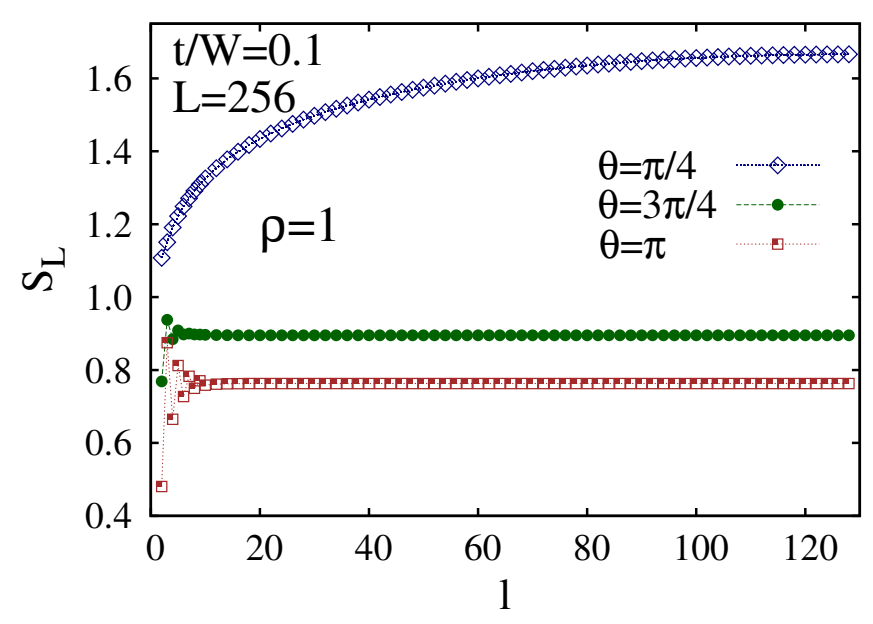

FIG. 7. The von Neumann block entropy $S_{L}(l)$ as a function of $l$ for a system with size $L=256, \rho=1$ and $t / W=0.1$. Here we consider three different values of statistical angle: $\theta=\pi / 4, \theta=3 \pi / 4$, and $\theta=\pi$. The statistical angle drives a quantum phase transition.

The density profile of the particles along a lattice of $L=120$ with $\theta=3 \pi / 4$ and $t / W=0.1$ is shown in the inset of Fig. 4. At the ends of the lattice, we note strong fluctuations due to the open boundary conditions considered in our study. Despite this, we obtain that at each site there is one particle, i. e. $\left\langle n_{i}\right\rangle=1$. This result, as well as the fact that there is a finite gap at the thermodynamic limit, allows us to conclude that the ground state is a Mott insulator, which is generated by the interplay of statistics and interactions, because for spinless and spinor bosons under local three-body interactions it is impossible to obtain a Mott insulator lobe with $\rho=1$. To distinguish the Mott regions from the superfluid ones, we draw vertical lines in Fig. 4 which shows the global density as a function of the chemical potential at the thermodynamic limit for $\theta=3 \pi / 4$ and two different values of the hopping $t / W=0.1$ and $t / W=0.4$. From this figure it is clear that a quantum phase transition from a Mott insulator to a superfluid phase will take place, and we observe that for both values of $t / W$ the compressibility $(\kappa=\partial \rho / \partial \mu)$ is always greater than zero, which indicates the absence of a first-order transition. Also, we obtained that the overall behavior of the global density as a function of $\mu / W$ is the same for both values of the hopping. However, the Mott gap is obviously larger at $t / W=0.4$ than at $t / W=0.1$. This unusual result can be understood by taking into account that for generating a Mott insulator state with $\rho=1$ under local three-body interactions, we need the interaction term to be important. For this, the quantum fluctuations must grow, which is caused by the hopping, and with the localization due to the statistics we obtain a larger effective interaction term, and finally the Mott gap increases with the hopping.

Up to this point, we know that for zero and small values of the statistics $(\theta \leq \pi / 4)$ there is not a Mott insula- tor state with a global density $\rho=1$ whatever the value of the three-body interaction and that at the pseudofermion limit $(\theta=\pi)$ there is a Mott insulator state in the absence of interactions. Also, our results away of the extreme limits suggest that both the statistics and the interaction are necessary to generate a Mott insulator state with one particle per site. To observe more clearly the effect of the three-body interaction, we temporarily change our energy unit to $t=1$ and express all quantities in terms of this. The global density as a function of the chemical potential $\mu / t$ appears in Fig. 5 where a statistical angle of $\theta=3 \pi / 4$ and different values of the local three-body interaction were considered. For global densities smaller than $\rho=1$, the before reported $S F_{0}$ superfluid $(\rho<0.34)$ and the paired $(\rho>0.34)$ phases were obtained [27], but the new fact observed is that the first phase and in some region of the second they do not depend of the three-body interaction, because there are few particles and the effective interaction is determinate by the statistics. We note that the effect of interactions is more striking from the global density $\rho=0.75$. After the above density the equation of state $\rho(\mu)$ depends strongly on the interaction, note that the existence or not of plateaus depends on the value of the local interaction. For small values of interaction $W / t=0.5$ and $W / t=1.0$ there is not a plateau at a global density $\rho=1$ indicating that there is not a Mott insulator state for these values (see Fig. 6(b)), also we can see that the scope of the curve increases suggesting a separation of phases (PS), after this a paired phase and Mott insulator state with $\rho=2$ were obtained. When the three-body interaction increases $(W / t=2.0)$ a plateau appears at the density $\rho=1$, where the evolution of the width of the plateau as a function of the latiice size is shown in Fig. 6(c), being clear that at the thermodynamic limit the width of the plateau is finite and the ground state is a Mott insulator. Keeping the statistical angle and the value of the interaction, we obtain that between the Mott insulator states for the integer densities there is a superfluid phase $\left(S F_{\pi}\right)$. From Fig. 5 we note that the ground state changes drastically as the three-body interaction increases, for non-integer densities the ground state goes from a paired phase to a superfluid one, at density $\rho=1$ a change from a gapless phase to a Mott insulator state takes place. Also, we can see that the width of the plateaus at the integer densities increases with the three-body interaction (see the inset of Fig. 5, where a zoom of the plateau at $\rho=1$ is shown for $W / t=2.0$, 5.0 , and 8.0 ). In conclusion for a system of anyons with a statistical angle $\theta=3 \pi / 4$ without interactions there is not a Mott insulator state with one particle per site, once the interaction is turn on, the Mott insulator state appears from a critical value of the interaction. Figs. 6(b) and 6(c) tell us that the critical value of the interaction is between $W / t=1.0$ and 2.0, where the width of the plateau vanishes and has a finite value respectively. The evolution of the width of the plateau as a function of the local interaction at the thermodynamic limit is shown in 


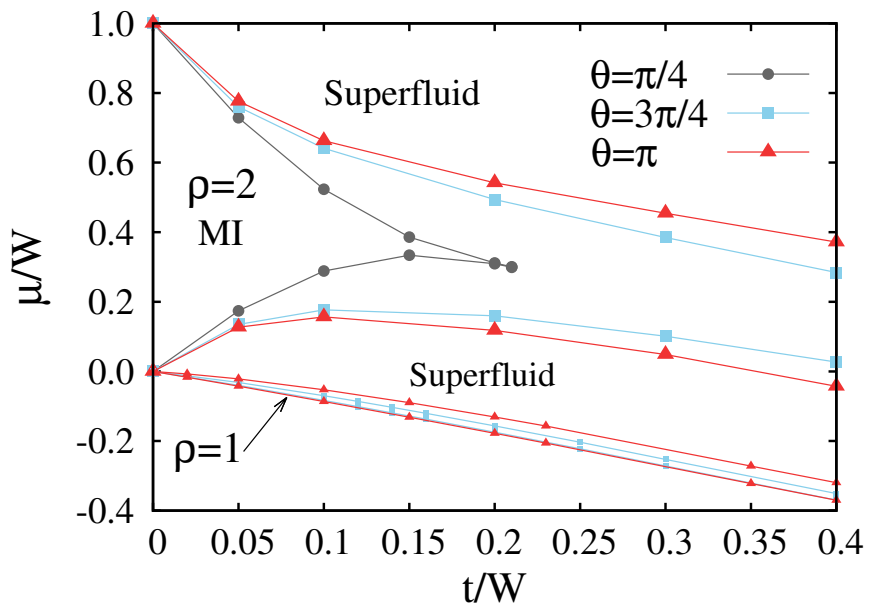

FIG. 8. Phase diagram of the anyon-Hubbard model with local three-body interactions for $\theta=\pi / 4,3 \pi / 4$, and $\pi$. Mott lobes and a superfluid phase were found, and their boundaries are marked by points that are extrapolations to the thermodynamic limit from DMRG data. The lines are visual guides.

Fig. 6] where two different regions are clear, the first one is a gapless region for small values of $W / t$ and a Mott insulator phase, where the gap increases monotonously with the three-body interaction. We estimate that the quantum critical point is $W_{c} / t=1.1$. It is expected that the critical three-body interaction will depend on the value of the statistical angle $\theta$.

Previously, we showed that the behavior of the block von Neumann entropy allows us to distinguish between critical and noncritical ground states, for instance superfluid and Mott insulator states. With respect to the appearance of insulator states for larger values of the statistical angle, we want to verify the above results by means of the calculation of block entropy (5). We present the results in Fig. 7 for three different values of the angle, $\theta=\pi / 4, \theta=3 \pi / 4$, and $\theta=\pi$, setting the global density at $\rho=1$, the lattice size at $L=256$, and the tunneling at $t / W=0.1$, where again and untill the end of the paper our unit of energy will be $W=1$. For $\theta=\pi / 4$, we observe that the entropy always increases until it diverges, which indicates that the system is in a superfluid phase. This is consistent with our previous results, in which a non-insulator region was found for this angle and this density (Fig. 21). On the other hand, the above behavior of the block entropy changes for larger values of $\theta$. Specifically, we observe that the block entropy increases very quickly exhibiting an oscillator behavior for small values of the block size, but the range increases with the angle. We cannot explain this oscillator behavior, which is due to the statistics. After the short oscillation, the block entropy remains constant, showing that the system has a finite correlation length. Hence the ground state is a Mott insulator one. Another interesting fact is that the overall value of the von Neumann block entropy diminishes as the statistical angle grows, which indicates

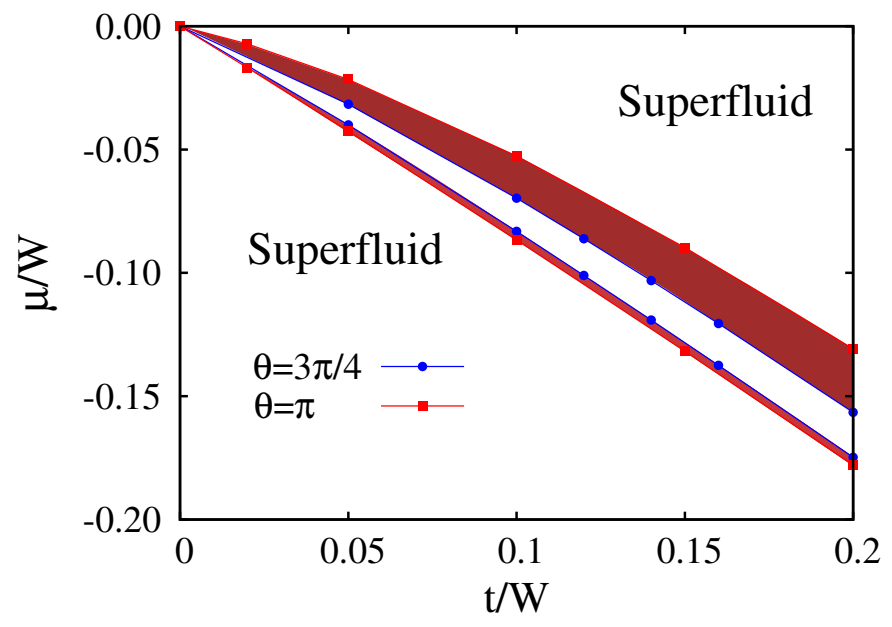

FIG. 9. The $\rho=1$ Mott lobe at the thermodynamic limit for a statistical angle $\theta=3 \pi / 4$ and $\theta=\pi$. The lines are visual guides.

that the entanglement decreases. The above discussion allows us to conclude that the statistical angle $\theta$ drives a quantum phase transition from a superfluid to a Mottinsulator phase with $\rho=1$.

We found that the statistics induce a Mott insulator region for larger values of the angle $\theta$. This fact suggests that the phase diagram changes with the statistical angle, and we have to calculate this for statistical angles greater than $\theta=\pi / 4$. In Fig. 8, we also draw the results for $\theta=\pi / 4$ shown in Fig. 2] in order to compare them with the new results obtained, $\theta=3 \pi / 4$ and $\theta=\pi$. We want to emphasize the absence of the $\rho=1$ Mott lobe for $\theta=\pi / 4$. At the atomic limit $(t / W=0)$, we observe that the Mott lobe regions are given by $\mu / W=$ $\rho-1$ regardless of the statistical angle; hence there is no Mott lobe with $\rho=1$ at this limit. For the nonzero hopping parameter, we see that the Mott lobe with $\rho=2$ decreases as $t / W$ grows, a fact that is maintained for any angle; however, it is clear that as the angle grows the statistics favor the localization of the particles, which is reflected in the increase of the Mott lobe area and the displacement of the critical point towards larger values. The phase diagram shows a much smaller $\rho=2$ Mott lobe than the anyon chain with two-body interactions. Also, we obtained a re-entrant behavior for the $\rho=2$ Mott lobe regardless of the statistical angle, i. e. for a fixed chemical potential value the ground state passes from a Mott insulator to a superfluid phase and then returns to the Mott insulator one. Note that this does not happen for the $\rho=1$ Mott lobe (see Fig. 9). The emergence and evolution of the $\rho=1$ Mott lobe as the hopping parameter increases is shown Fig. 9 for two different angles $\theta=3 \pi / 4$ and $\theta=\pi$, where a zoom in has been made. From this it is clear that if the statistics increase, the localization grows. Note that for negative constant values of the chemical potential, the ground state is a superfluid with density lower than one. As the kinetic 


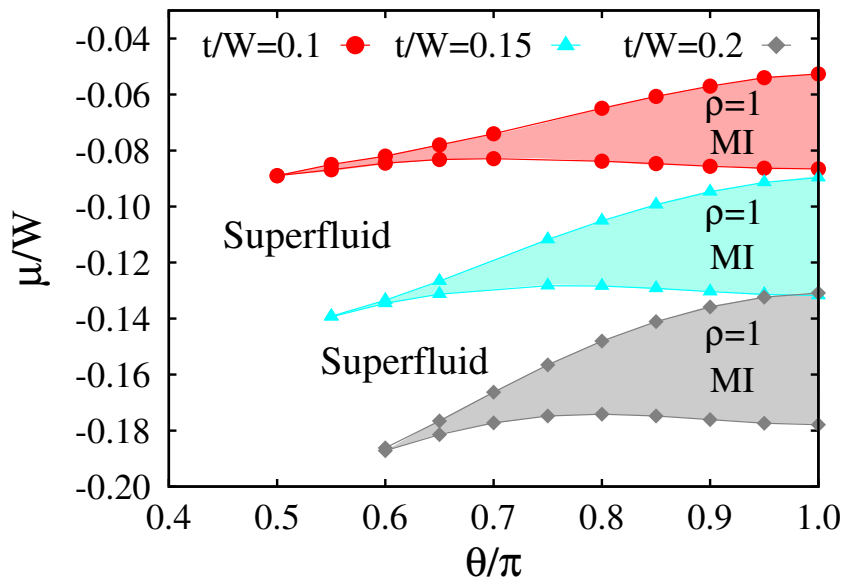

FIG. 10. Phase diagram of the anyon-Hubbard model with local three-body interactions in the plane $(\mu / W, \theta / \pi)$. We consider three different hopping parameters $t / W=0.1,0.15$ and 0.2 . The points are extrapolations to the thermodynamic limit from DMRG data and the lines are visual guides. MI means Mott insulator regions.

energy increases, it goes to a Mott insulator state with $\rho=1$ and finally returns to a superfluid state, but with a density greater than one. From this figure, it is clear that the Mott lobe area increases with the hopping.

We showed that the statistical angle drives a quantum phase transition from a superfluid state to a Mott insulator one in the range of $\theta=\pi / 4-3 \pi / 4$ for a fixed hopping parameter $t / W=0.1$ and global density $\rho=1$ (see Fig. 17). However a pending task is to explore the ground state phase diagram as a function of the statistical angle, which is shown in Fig. 10 for three different values of the hopping parameter, $t / W=0.1,0.15$, and 0.20 . We obtain that there is a critical value of the angle at which the Mott insulator phase appears, and this critical value moves to greater values as the hopping grows. A reentrance phase transition was observed, since the hole excitation energy curve exhibits a maximum. Hence at some suitable constant chemical potential, the model displays a sequence of quantum phase transitions between the Mott insulator and the superfluid phases. In the sequence (from left to right), we have a change from a superfluid region with $\rho>1$ to a Mott insulator one with $\rho=1$ and then again to an superfluid region $(\rho<1)$, and finally the system remains in an insulator state. Finally, we note that for a fixed angle, the Mott lobe area increases with the tunneling. Note that the shape of the Mott lobes around the critical point is not elongated like others shown in Fig. 2 or others reported previously, which can indicate that this quantum phase transition is not of the Kosterlitz-Thouless type.

It is well known in the literature that using the gap vanishing point to determine the critical point related to a quantum phase transition gives us poor results and that some measures of the entanglement can help us in this task. Precisely, it has been shown that for models like the

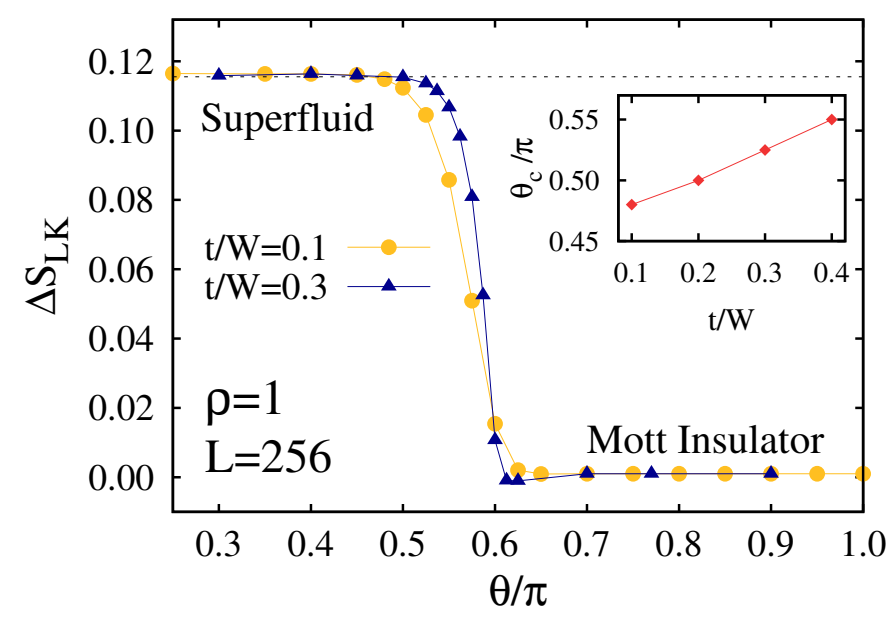

FIG. 11. The estimator $\Delta S_{L K}$ as a function of angle $\theta$ for $t / W=0.1$ and $t / W=0.3$. Here we fixed $\mathrm{L}=256$ and $\rho=1$. The points are DMRG data and the lines are a visual guide. Inset: Evolution of the critical angle $\theta_{c}$ with the hopping parameter.

Bose-Hubbard one, the estimator based on the von Neumann block entropy $\Delta S_{L K}(L)=S_{L}(L / 2)-S_{L / 2}(L / 4)$ proposed by Läuchli and Kollath leads to better results [50]. According to above definition and the expression Eq. (51), we obtain:

$$
\Delta S_{L K}(L)= \begin{cases}\frac{c}{6} \ln [2], & \theta \leq \theta_{c}, \\ 0, & \theta>\theta_{c},\end{cases}
$$

$\theta_{c}$ being the critical angle.

In Fig. 11, the evolution of the estimator $\Delta S_{L K}$ with the statistical angle is shown for an anyon chain with $\rho=1$, and $L=256$. When $\theta=0$, the ground state is a superfluid, because the $\rho=1$ Mott insulator lobe does not exist for bosons under local three-body interactions. Therefore, the estimator will be equal to $\ln (2) / 6$, and it remains at this point for non-zero values of the statistical angle, indicating that there is a range of values of $\theta$ for which the ground state is a superfluid. However, from a given critical angle the estimator collapses to zero within a short range. After that, the estimator remains constant at zero, indicating that the ground state is now a Mott insulator one. Regardless of the hopping parameter, we see that the estimator clearly shows us the quantum phase transition from a superfluid to a Mott insulator state. Note that the superfluid region becomes greater and the shape of the curve becomes sharper as the hopping increases, although at the thermodynamic limit the shape of the curve will be a step function, according to the expression Eq. (6). The critical point corresponds to the greatest value of $\theta$, for which the estimator is equal to $\ln (2) / 6$, and we obtain that the position of the critical angle moves to greater values as the hopping grows (see inset of Fig. 11). We use the above procedure to determinate the critical points due to we do not know a priori the order of the transition and that the phase diagram 


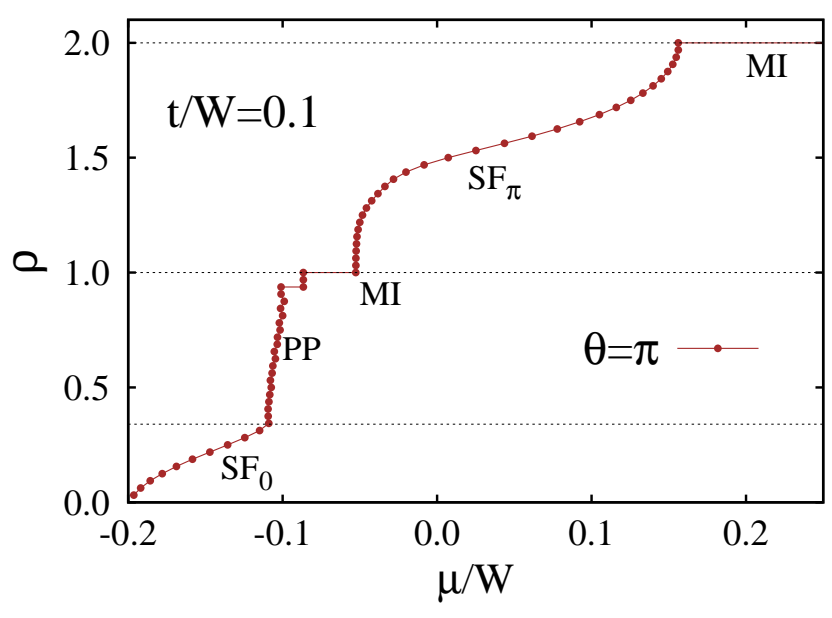

FIG. 12. Equation of state $\rho=\rho(\mu)$ for anyons under a finite three-body interaction $t / W=0.1$ in the pseudo-fermion limit $(\theta=\pi)$. The points are DMRG data and the lines are a visual guide.

show in Fig. 10 suggest that the transition is not the Kosterlitz-Thouless type, which prevents us from using the gap-scaling analyses, which led to the most accurate determination of the critical point in Bose-Hubbard model [47, 48]. An alternative way to estimate the critical points is calculating the Luttinger-liquid parameter, which is a very expensive numerical way to determinate the critical points [49].

The pseudo-fermion limit $(\theta=\pi)$ of the anyonHubbard model attracted attention and is full of surprises, for instance Greschner and Santos found a Mott insulator phase with one particle per site in absence of interactions and diverse superfluid phases for non-integer densities [27]. Recently Zhang and co-workers considered a non-zero finite two-body interaction between the anyons and observed that the ground state for noninteger densities is very rich, showing transitions from superfluids to a separation phase or paired phase for densities lower or greather than $\rho=1$, respectively [31]. Motivate by these results, we show in Fig. 12 the equation of state $\rho=\rho(\mu)$ at the pseudo-fermion limit for a finite three-body interaction $t / W=0.1$. For densities lower than $\rho=0.34$, we obtained a superfluid phase $S F_{0}$, after that the scope of the curve changes and a paired phase appears, result that diverge of the result for finite twobody interactions where a separation phase was found, this divergence is expected since the effective interaction (statistics plus three-body potential) is weaker in our case than in the two-body calculation. Between the Mott insulator plateaus, we obtained a superfluid phase $S F_{\pi}$ in the whole region, whereas for a finite two-body interaction a quantum phase transition from a superfluid phase $S F_{\pi}$ to paired phase was reported. We see that our re- sults for a finite three-body interaction are more closer to the non-interacting case than the finite two-body interactions, due to the smaller effective interactions.

\section{Conclusions}

We studied the interplay of multi-body interactions and the statistics in an anyon-Hubbard model, which was mapped to a modified Bose-Hubbard model by means of a fractional Jordan-Wigner transformation. This lastnamed model was explored using the density matrix renormalization group method, and we found that the model exhibits gapless and gapped regions in the phase diagram. The expression $\mu / W=\rho-1$ determines the width of the Mott lobes at the atomic limit, regardless of the statistics. As in the spinless and spinor boson cases, we obtain that for small statistical angles there is no Mott insulator region with density $\rho=1$ when local three-body interactions are considered. However the above Mott insulator state emerges as the statistics grow, from a critical value of the local interaction, which is $W / t=1.1$ for $\theta=3 \pi / 4$, this fact being one of the main findings of this study.

Away from the extreme limits, we found that together the statistics and the local three-body interaction drive a quantum phase transition from a gapless phase to a Mott insulator one when the density is $\rho=1$. This quantum phase transition was clearly identified by means of the von Neumann block entropy and the estimator proposed by Läuchli and Kollath [50]. Using the latter to estimate the critical angles, we see that the hopping parameter moves the critical angles to greater values.

Although a complete study of the ground state for noninteger densities is out scope of this paper, we found that the equation of state $\rho=\rho(\mu)$ at the pseudo-fermion limit for a finite three-body interaction is closer to the non-interacting case, due to the weaker effective interactions. Away from the pseudo-fermion limit, we observe diverse gapless phases, such as: superfluid $S F_{0}$, paired phase, superfluid $S F_{\pi}$ and a possible separation phase. The quantum phase transitions between the above gapless phases and their evolution with the local three-body interactions for different statistical angles will be explore in a future study.

\section{ACKNOWLEDGMENTS}

We thank Sebastian Greschner for useful comments. All authors are thankful for the support of DIEB- Universidad Nacional de Colombia and COLCIENCIAS (grant No. FP44842-057-2015). Silva-Valencia and Franco are grateful for the hospitality of the ICTP, where part of this work was done. 
[1] J. M. Leinaas and J. Myrheim, Il Nuovo Cimento B 37, 1 (1977).

[2] F. Wilckzek, Phys. Rev. Lett. 49, 957 (1982).

[3] F. D. M. Haldane, Phys. Rev. Lett. 67, 937 (1991).

[4] D. C. Tsui, H. Stormer, and J. Gossard, Phys. Rev. Lett. 48, 1559 (1982).

[5] R. B. Laughlin, Phys. Rev. Lett. 50, 1395 (1983).

[6] B. I. Halperin, Phys. Rev. Lett. 52, 1583 (1984).

[7] F. E. Camino, W. Zhou, and V. J. Goldman, Phys. Rev. B 72, 075342 (2005).

[8] A. Y. Kitaev, Ann. Phys. 303, 2 (2003).

[9] C. Nayak, S. H. Simon, A. Stern, M. Freedman, and S. D. Sarma, Rev. Mod. Phys. 80, 1083 (2008).

[10] J. Alicea, Y. Oreg, G. Rafael, F. von Oppen, and M. P. A. Fisher, Nat. Phys. 7, 412 (2011).

[11] A. Kundu, Phys. Rev Lett. 83, 1275 (1999).

[12] A. Osterloh, L. Amico, and U. Eckern, J. Phys. A 33, L487 (2000).

[13] M. Batchelor, X.-W. Guan, and N. Oelkers, Phys. Rev Lett. 96, 210402 (2006).

[14] P. Calabrese and M. Mintchev, Phys. Rev. B 75, 233104 (2007).

[15] Y. Hao and S. Chen, Phys. Rev. A 86, 043631 (2012).

[16] O. I. Patu, J. Stat. Mech. 2015, P01004 (2015).

[17] N. T. Zinner, Phys. Rev. A 92, 063634 (2015).

[18] C. Vitoriano, Phys. Rev. Lett. 102, 146404 (2009).

[19] M. D. Girardeau, Phys. Rev. Lett. 97, 100402 (2006).

[20] B. Paredes, P. Fedichev, J. I. Cirac, and P. Zoller, Phys. Rev. Lett. 87, 010402 (2001).

[21] L.-M. Duan, E. Demler, and M. D. Lukin, Phys. Rev. Lett. 91, 090402 (2003).

[22] L. Jiang, G. K. Brennen, A. V. Gorshkov, K. Hammerer, M. Hafezi, E. Demler, M. D. Lukin, and P. Zoller, Nat. Phys. 4, 482 (2008).

[23] M. Aguado, G. K. Brennen, F. Verstraete, and J. I. Cirac, Phys. Rev. Lett. 101, 260501 (2008).

[24] S. Longhi and G. D. Valle, Optics Letters 37, 2160 (2012).

[25] Y. Zhang, G. J. Sreejith, and J. K. Jain, Phys. Rev. B 92, 075116 (2015).

[26] T. Keilmann, S. Lanzmich, I. McCulloch, and M. Roncaglia, Nat. Commun. 2, 361 (2011).

[27] S. Greschner and L. Santos, Phys. Rev. Lett. 115, 053002 (2015).

[28] L. Cardarelli, S. Greschner, and L. Santos, Phys. Rev. A 94, 023615 (2016).

[29] C. Sträter, S. C. L. Srivastava, and A. Eckardt, Phys. Rev. Lett. 117, 205303 (2016).
[30] J. Arcila-Forero, R. Franco, and J. Silva-Valencia, Phys. Rev. A 94, 013611 (2016).

[31] W. Zhang, S. Greschner, E. Fan, T. C. Scott, and Y. Zhang, Phys. Rev. A 95, 053614 (2017).

[32] G. Tang, S. Eggert, and A. Pelster, New J. Phys. 17, 123016 (2015).

[33] G. Marmorini, M. Pepe, and P. Calabrese, J. Stat. Mech. 2016, 073106 (2016).

[34] F. Lange, S. Ejima, and H. Fehske, Phys. Rev. Lett. 118, 120401 (2017).

[35] E. Duchon, Y. L. Loh, and N. Trivedi, (2013), ArXiv:1311.0543.

[36] M. Greiner et al., Nature 419, 51 (2002).

[37] S. Will, T. Best, U. Schneider, L. Hackermüller, D.-S. Lühmann, and I. Bloch, Nature 465, 197 (2010).

[38] R. Ma, M. E. Tai, P. M. Preiss, W. S. Bakr, J. Simon, and M. Greiner, Phys. Rev. Lett. 107, 095301 (2011).

[39] J. Silva-Valencia and A. M. C. Souza, Phys. Rev. A 84, 065601 (2011).

[40] J. Silva-Valencia and A. M. C. Souza, Eur. Phys. J. B 85, 161 (2012).

[41] A. J. Daley and J. Simon, Phys. Rev. A 89, 053619 (2014).

[42] T. Sowiński, Cent. Eur. J. Phys. 12, 473 (2014).

[43] H.-N. Dai, B. Yang, A. Reingruber, H. Sun, X.-F. Xu, Y.-A. Chen, Z.-S. Yuan, and J.-W. Pan, Nat. Phys. 12, 783 (2017).

[44] L. Amico, R. Fazio, A. Osterloh, and V. Vedral, Rev. Mod. Phys. 80, 517 (2008).

[45] R. Islam, R. Ma, P. M. Preiss, M. E. Tai, A. Lukin, M. Rispoli, and M. Greiner, Nature 528, 77 (2015).

[46] P. Calabrese and J. Cardy, J. Stat. Mech. 2004, P06002 (2004).

[47] J. Carrasquilla, S. R. Manmana, and M. Rigol, Phys. Rev. A 87, 043606 (2013).

[48] M. Dalmonte, J. Carrasquilla, L. Taddia, E. Ercolessi, and M. Rigol, Phys. Rev. B 91, 165136 (2015).

[49] R. T. Clay, A. W. Sandvik, and D. K. Campbell, Phys. Rev. B 59, 4665 (1999).

[50] A. M. Läuchli and C. Kollath, J. Stat. Mech. 2008, P05018 (2008).

[51] Ö. Legeza, J. Roder, and B. A. Hess, Phys. Rev. B 67, 125114 (2003).

[52] C. A. Avila, R. Franco, A. M. C. Souza, M. S. Figueira, and J. Silva-Valencia, Phys. Lett. A 378, 3233 (2014). 